\title{
THE INFRA-RED EMISSION SPECTRUM OF THE MERCURY ARC.
}

By W. W. Coblentz and W. C. Geer.

$T$ HE present investigation of the radiation from the mercury arc was undertaken to account for the high efficiency ${ }^{1}$ of the Arons lamp.

The spectrum was produced by means of a mirror spectrometer, and a rock salt prism, while a Nichols radiometer was used to measure the distribution of energy. The whole is shown in Fig. I in which $N$ is the source, $S$ the shutter, $P$ the rock salt prism and $R$ the radiometer. A full description of the adjustment and calibration of the apparatus is given elsewhere ${ }^{2}$ and need not be mentioned here.

The Mercury Arc.

The arc was formed in a vacuum tube

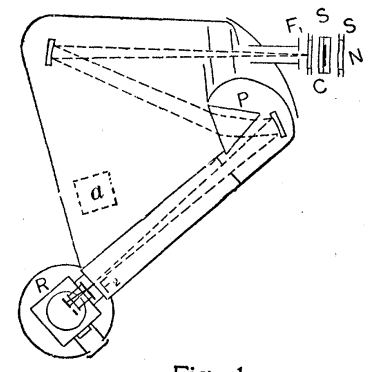

Fig. 1. which was a modification of that first described by Arons. ${ }^{3}$ The

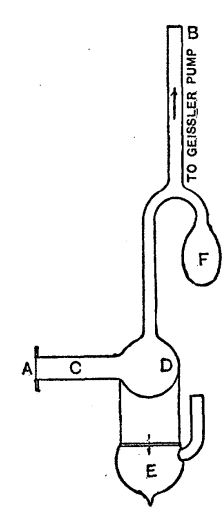

Fig. 2. vertex of the arc was used as a source of radiation. For this purpose a side tube, having a window of fluorite or rock salt, was placed at right angles to the plane of the arc.

The side elevation is shown in Fig. 2. The diameter of $C$ was I cm., that of $D 2.5 \mathrm{~cm}$., with the other parts in proportion. Throughout the work the lamp was connected to a Geissler pump and when in use the stopcock at $B$ was closed. The whole was enclosed in a tin box containing water with the side tube, $C$, projecting through a water-tight sleeve. A fluorite window was at $A$ for wave-

1 Geer, Physical Review, Vol. XVI., No. 2, 1903.

2 Coblentz, Physical Review, Vol. XVI., Nos. I and 2, I903.

3 Arons, Wied. Ann., 47, 767, r892. 
lengths up to $6 \mu$, while a window of rock salt was employed from 6 to $9 \mu$.

The mercury was purified by running it through a separatory funnel containing mercury, on the surface of which was a layer of mercurous sulphate and concentrated sulphuric acid. The mercury treated in this way was some that had been standing in contact with the cleaning agent for several weeks. By this means it was freed from moisture and metallic impurities. It left no weighable residue upon evaporation.

During the exhaustion the mercury was continually flowed from one leg of the lamp to the other to free it from all traces of air. Throughout the investigation the lamp was pumped out at frequent intervals in order to be certain that the vacuum was maintained. On starting the arc the vacuum was always less than $0.03 \mathrm{~mm}$.

A direct current of six ampères was used throughout the work. The water in the tank varied from $50^{\circ}$ to $80^{\circ}$. It was allowed to become warm since it produced a larger arc. Larger currents were not used for fear of breaking the lamp.

\section{Curves and Results.}

It was found that the energy radiated consists of a series of emission bands at I $\mu$ and $5 \mu$ with a slight indication of a band at $3 \mu$. Nowhere in the spectrum was the intensity of the radiation from the arc very great. This indicates a low temperature like that of gases in vacuum tubes, ${ }^{1}$ although Arons ${ }^{2}$ has computed a value of several thousand degrees.

The distribution of the maxima is best shown by the curves in Fig. 3. For the region at $\mathrm{I} \mu$ the mean of several readings has been plotted, while beyond $4 \mu$ individual readings are given.

In Table I. a typical set of readings is given for the region from $\lambda=0.92$ to $\lambda=2.0 \mu$. It shows that the variation in the individual

${ }^{1}$ Salisbury first observed the low temperature of such gases. Phil. Mag. (4), 45, p. 241, 1873.

E. Wiedemann, Calorimeter, Wied. Ann., 6, p. 298, r878; 10, p. 202, r 880.

Paalzow Weesen, Wied. Ann., 56, p. 276, 1895.

Warburg, Theory, Wied. Ann., 54, p. 265, 1895.

Wood, Bolometer, Wied. Ann., 59, p. 238, I896.

2 Arons, Wied. Ann., 58, 1896. 
readings is not great, when one considers the smallness of the deflections.

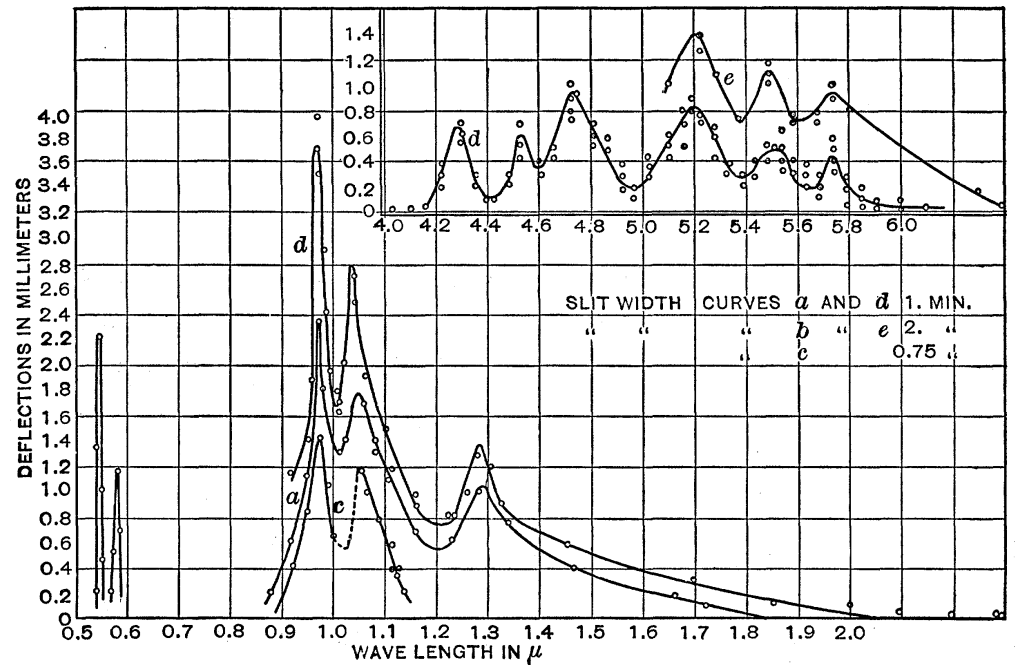

Fig. 3.

TABle I.

Deflections in Millimeters. Wave-lengths in $\mu$.

\begin{tabular}{|c|c|c|c|c|c|c|c|c|c|c|c|c|c|c|c|}
\hline$\lambda$ & .92 & .95 & .98 & I.OI & 1.02 & 1.06 & $x .08$ & I.IO & 1.16 & 1.23 & $\mathrm{r} .28$ & I.34 & $x .46$ & $x .72$ & 2.03 \\
\hline \multirow{4}{*}{ 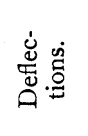 } & 0.5 & 0.9 & 1.7 & 1.5 & 1.5 & 1.7 & 1.3 & 1.2 & 0.8 & 0.7 & 1.0 & 0.8 & .03 & 00 & 00 \\
\hline & 0.4 & 1.4 & 2.0 & 1.2 & 1.4 & 1.7 & 1.4 & 1.3 & 0.6 & 0.5 & 1.1 & 0.9 & .05 & 00 & 00 \\
\hline & 0.6 & 1.0 & 1.8 & 1.1 & 1.3 & 1.8 & & 1.2 & & & 1.0 & & & .02 & 00 \\
\hline & 0.8 & & 1.8 & 1.4 & & 1.6 & & 1.1 & & & 0.8 & & & & \\
\hline Mean, & 0.5 & 1.1 & 1.8 & 1.3 & 1.4 & 1.7 & 1.4 & 1.2 & 0.7 & 0.6 & 1.0 & 0.9 & .04 & 00 & 00 \\
\hline
\end{tabular}

\begin{tabular}{|c|c|c|c|c|c|c|c|c|c|c|c|c|c|c|c|}
\hline$\lambda$ & .92 & .95 & .96 & 97 & .98 & .995 & I.OI & 1.02 & $\mathrm{I} .04$ & I.06 & 1.16 & $\mathrm{I} .26$ & 1.28 & 1.32 & I.34 \\
\hline \multirow{4}{*}{ 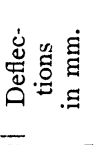 } & 1.2 & 1.4 & 1.6 & 3.8 & 3.0 & 1.9 & 1.6 & \multirow[t]{4}{*}{2.0} & 2.2 & 1.6 & 0.8 & 0.8 & 1.1 & 0.9 & 0.7 \\
\hline & 1.1 & & 1.9 & 3.5 & 2.8 & 2.0 & 1.8 & & 2.4 & 1.7 & 1.0 & 0.5 & 1.0 & 0.9 & 0.8 \\
\hline & & & & 4.2 & & & & & 2.7 & 1.9 & & & 1.3 & & \\
\hline & & & & & & & & & 2.5 & & & & 1.3 & & \\
\hline Mean, & 1.1 & 1.4 & 1.8 & 3.7 & 2.9 & 2.0 & 1.7 & 2.0 & 2.5 & 1.7 & 0.9 & 0.6 & 1.2 & 0.9 & 0.7 \\
\hline
\end{tabular}

The difficulties in observing the deflections were extreme on account of the weakness of the radiation. These small deflections are not due to lack of sensitiveness of the radiometer, for, under like 
conditions, a cylindrical acetylene flame gave a deflection of $50 \mathrm{~cm}$. at the maximum part of its spectrum, while the $\mathrm{CO}_{2}$ emission band of a bunsen acetylene burner at $4.4 \mu$ threw the deflection clear off the scale.

Although the deflections were small they were trustworthy. It should be remembered that the radiometer at the sensitiveness used was unusually stable. The mirror suspension was not the lightest, so that earth tremors rarely caused annoyance. No readings were made when such vibrations were noticeable. The period of the radiometer was about thirty seconds. In order to reduce the errors due to temperature changes it was necessary to shorten the period at a sacrifice of the sensitiveness of the instrument. The radiometer was packed in wool, in a tin box, and the zero shift, due to changes of temperature, for single readings was generally only o. I to $0.2 \mathrm{~mm}$., while often it was not to be noticed at all. The whole apparatus stood in an inner room and the deflections were observed through a hole in the wall. When there was no disturbance in the room the zero shift was always extremely small. On exceptional days-not for this work, however-the zero shift could not be detected during ten minutes, on actual count, while in four hours it amounted to only $1.6 \mathrm{~cm}$.

In the first exploration (slit $\mathrm{I} \mathrm{mm}$. in width), up to $6 \mu$, the apparatus was not so sensitive and no radiation from the hot walls of the tube could be detected. But when the fluorite window was replaced by one of rocksalt, and an increased sensitiveness of the radiometer was combined with a slit of $2 \mathrm{~mm}$. width, the hot glass radiation was perceptible beyond $5 \mu$. Consequently, for each setting of the spectrometer the radiation from the hot glass was measured and deducted. For the latter measurement the shutter at the window was raised at the instant that the arc was extinguished. It was found that beyond $6 \mu$ up to $9 \mu$, which was so far as the exploration extended, the average deflections for the hot glass were as large as those due to the arc.

The spectrum was explored at every minute of the spectrometer circle up to $9 \mu$, while in certain regions readings were made at every $2 \mathrm{O}^{\prime \prime}$. Consequently, this required several days and several maxima of the curves were tested each day in order to determine 
their relative heights. This variation of height, due to the changes in the sensitiveness of the instrument, is shown in Fig. 3. The best values for the relative heights is shown in the curve $b$. In Fig. 4 the complete curve is given. The deposition of mercury on the window was one source of annoyance and at times vitiated the readings. On three different days observations were made on the region from $2 \mu$ to $4 \mu$, but no radiation could be detected except at about $3 \mu$ where there was a slight deflection at times. This is of interest in what follows.

The reflecting effect of the glass walls is not known. Since glass becomes opaque at $3 \mu$ it will be noticed that all the energy of

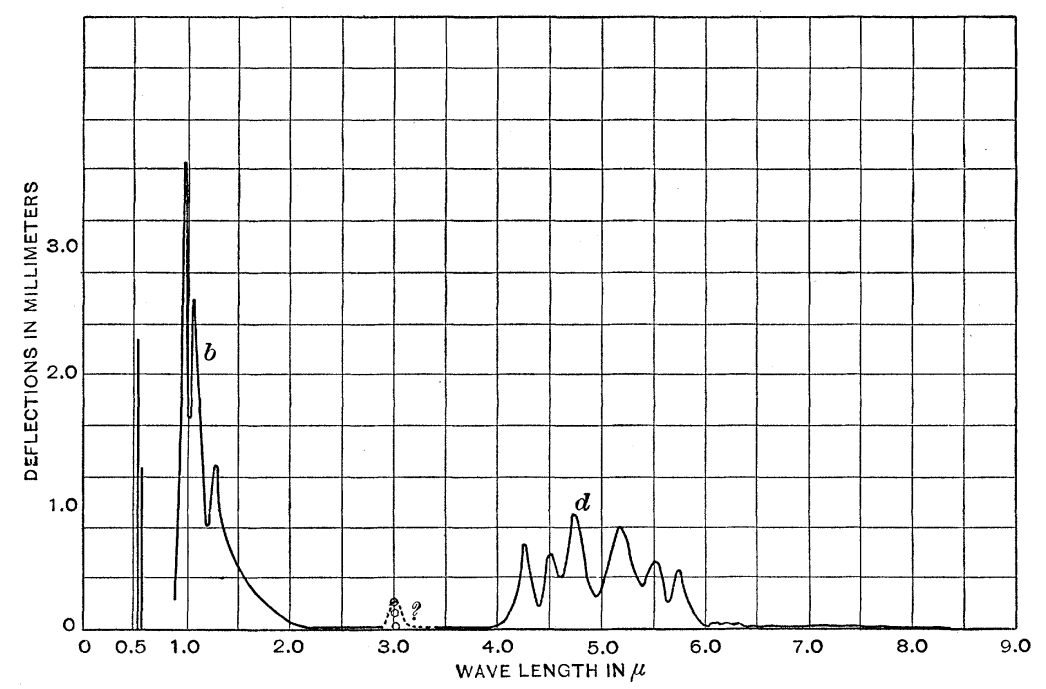

Fig. 4.

greater wave-length is absorbed by the glass in a lamp of the form described by Arons. Thus the apparent radiant efficiency of the arc would be greater than the true efficiency. This accounts in part for the unusually high values found in the previous investigation. ${ }^{1}$

The width of the spectrum covered by the radiometer vane at I $\mu$ was about . I $5 \mu$. The error at I $\mu$ is less than .or $\mu$ while at $4 \mu$ it s perhaps $.03 \mu$.

\footnotetext{
${ }^{1}$ Geer, l. c.
} 
Addendum on Spectral Series.

After the work had been completed, the senior writer repeated some of the observations to find the bearing of the emission maxima on laws for spectral series. Also to learn whether another line exists between I.06 and I.I $2 \mu$ where the curve is very asymmetrical. It was found that the mean of a great many readings was very nearly the same for $\lambda=1.08$ and I.10 $\mu$, and if another line exists it is very weak.

From their work on the elements of the second group of Mendeleeff's classification, viz., $\mathrm{Mg}, \mathrm{Ca}, \mathrm{Sr}, \mathrm{Ba}$; and $\mathrm{Cd}, \mathrm{Zn}, \mathrm{Hg}$, Kayser and Runge ${ }^{1}$ have found that there are groups of lines, triplets, distributed throughout the visible spectrum, whose position can be expressed by an empirical formula of the form

$$
\mathrm{I} / \lambda=A+B n^{-2}+C n^{-4}
$$

where $\mathrm{I} / \lambda$ is a function of the frequency and $n$ represents the successive integral numbers. They have also found that the difference between the values of $\mathrm{I} / \lambda$ for the members of any triplet is constant for any given element. In the case of mercury this difference is

$$
\begin{gathered}
v_{1}=\left\{\begin{array}{l}
(45.84 .6) \\
4630
\end{array}\right. \\
v_{2}=\mathrm{I} 760.0 .
\end{gathered}
$$

By means of their formula, for $n=3$, they predicted a triplet at $\lambda=$ $.9497 \mu, .6595 \mu, .5906 \mu$. But they add that these lines may lie still farther toward the infra-red. This follows from the fact that the wave number, $\mathrm{I} / \lambda$ in negative powers of $n^{2}$ forms a rapidly converging series, and three terms were sufficient for their work. Now the triplet, $n=3$, of the first subordinate series, falls in the infra-red and the computed values of $\lambda$ are obtained through wide extrapolation. They add that the formulæ require more terms with smaller powers of $n$, when $n$ is less than 3 . Hence they expected to find that for cæsium the predicted infra-red lines would be farther out by $.02 \mu$.

From the curves in Fig. 3 it will be seen that maxima occur at $.97, \mathrm{I} .045, \mathrm{I} .285,4.28,4.53,4.73,5.20,5.50$ and $5.73 \mu$. It

1 Kayser \& Runge, Die Sprecten der Elemente, IV.; Wied. Ann., 4I, 302, I89o. 
will be observed that the one at $.97 \mu$ agrees remarkably well with the predicted value $.9497 \mu$, especially when one remembers that Kayser and Runge expected the latter value to be larger. The fact that the radiometer failed to detect the other two lines is no reason for considering that this is not the third. Still more interesting is the fact that the difference between $I / \lambda$ for the maxima at $I .045 \mu$ and I. $285 \mu\left(v_{2}=1787\right)$ are unusually close to those in the visible spectrum, that is, they appear to belong to another triplet, of which the third component lies at $3.05 \mu$. The latter is obtained by computation, using the value $v_{1}=4630$. Since at $3.0 \mu$ the radiometer showed slight deflections at times which were recorded as questionable, and since in all other parts of the spectrum from 2 to $4 \mu$ no such effect could be detected, one is led to believe that the deflections observed as $3 \mu$ were real. The great variation in the intensity of these lines may be due, in part, to the fact that the slit subtends different angles in the two regions, and that the suspected line at $3 \mu$ is isolated while the others occur in a group, each one of which is influenced by those adjoining it.

In Table II. are given the observed maxima and the products obtained by multiplying each one by the arbitrary factor 4.4 . According to Kayser and Runge the first, second and third components of a sharp or diffuse series of triplets form, in each element, a line series. Whether the maxima at 4 to $6 \mu$ are thus related to those at I $\mu$ has not been determined, but the coincidence, when the arbitrary factor 4.4 is used, is very striking.

TABLE II.

\begin{tabular}{|c|c|c|c|c|}
\hline \multicolumn{2}{|c|}{$A^{\circ}$. U. from Kayser and Runge. } & \multirow{2}{*}{ 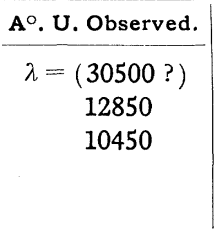 } & \multirow{2}{*}{$\left.\begin{array}{l}10^{8} \div \lambda \\
7783 \\
9570\end{array}\right\}$} & \multirow{2}{*}{$\begin{array}{l}\text { Difference. } \\
v_{2}=1787\end{array}$} \\
\hline $\begin{array}{r}\lambda=3007.02 \\
2642.70 \\
2524.80 \\
3680.74 \\
3144.61\end{array}$ & $\begin{array}{l}v_{1}=4584.6 \\
v_{2}=1767.0 \\
v_{1}=4631.8\end{array}$ & & & \\
\hline \multicolumn{2}{|c|}{ Maxima. } & Maxima $\times 4.4$ & & Observed. \\
\hline & & $\begin{array}{l}4.27 \\
4.56 \\
5.68\end{array}$ & & $\begin{array}{l}4.28 \\
4.53 \\
5.73\end{array}$ \\
\hline
\end{tabular}


The unavoidable errors in drawing the curves account, in part, for the variations between the predicted and the actual positions of the maxima. Since most of the curves were drawn prior to the consideration of their bearing on the question of spectral series the position of the maxima is not biased by the latter. An inspection of Fig. 2 shows that, through the few points observed, curves can be drawn which have different trends, especially when they are asymmetrical. For this region a prism of greater dispersing power, e. $g$., carbon disulphide, would have increased the accuracy of the work.

\section{Conclusion.}

From the foregoing investigation it is seen that the infra-red spectrum of the mercury arc comprises several well-defined bands with perfectly definite maxima. These maxima occur at the following wave-lengths: $0.97,1.045,1.285,4.28,4.53,4.73,5.20,5.50$ and $5.73 \mu$.

The presence of the bands of wave-lengths greater than $4 \mu$ indicates that the true radiant efficiency of the arc is lower than that previously found.

The maxima of the curves at $.97, \mathrm{I} .045$ and $\mathrm{I} .285 \mu$ follow quite closely the law of spectral series as developed by Kayser and Runge

CORNELL UNIVERSITY, November, 1902. 